\title{
10 Auswahl der Zeitschrift
}

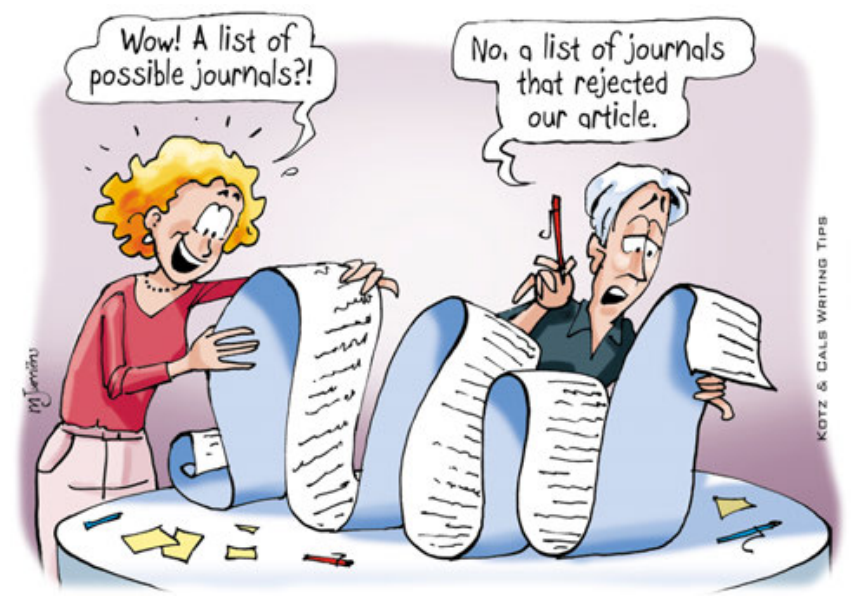

Tipp 10 - Auswahl der Zeitschrift: erstelle eine Liste potentieller Zeitschriften!

\section{Was Sie wissen sollten}

Mit Ihrem wissenschaftlichen Artikel möchten Sie eine Geschichte zu erzählen aber wer soll Ihnen dabei am liebsten zuhören? Ihr Publikum wird in großem Maße von der Zeitschrift abhängen, in der Sie Ihren Artikel veröffentlichen. Allein in PubMed sind derzeit etwa 30 Millionen Artikel aus 7.000 wissenschaftlichen Fachzeitschriften gelistet. Daraus kann man schlussfolgern, dass Sie mit etwas Ausdauer Ihren Artikel irgendwann irgendwo unterbringen werden. Die Wahl des richtigen Zuhauses will allerdings wohl überlegt sein.

Abhängig vom Prestige einer Zeitschrift, deren Platzbeschränkung und $\mathrm{Zu}$ strom von Neueinreichungen, variieren die Annahmeraten von Artikeln zwischen $10 \%$ und $80 \%$. Oftmals wird das Prestige einer Zeitschrift von deren Impact-Faktor abgeleitet. Dieser wird aus der Relation zwischen der Anzahl von Artikeln, die in den vorangegangenen zwei Jahren in der entsprechenden Zeitschrift veröffentlicht wurden (Nenner), und der Häufigkeit mit der diese von anderen Zeitschriften zitiert wurden (Zähler) jährlich neu berechnet. Über Sinn und Unsinn des Impact-Faktors wird seit langem heftig diskutiert, und dennoch wird dieser oftmals als Maßstab für die relative Bedeutung einer Zeitschrift angewandt. In den sogenannten Journal Citation Reports findet man die aktuellen 
Impact-Faktoren aller Zeitschriften und kann sich, falls gewünscht, die Rangfolge von Zeitschriften innerhalb des eigenen Fachgebietes anzeigen lassen.

Es gibt mittlerweile viele rein elektronische Zeitschriften, die ausschließlich im Internet erscheinen und ohne gedruckte Papierversion auskommen. Vorteile dieses Modells liegen darin, dass es keine Platzbeschränkung gibt (sowohl in der Anzahl der Artikel pro Jahr als auch in der Länge der jeweiligen Artikel), und dass jeder Artikel sehr schnell nach Annahme einzeln veröffentlicht werden kann, da Jahrgangsausgaben entfallen (dieses Prinzip nennt sich ,articlebased publishing“).

Immer mehr Zeitschriften sind zudem ganz oder teilweise „open access“ zugänglich. Dies beinhaltet einen uneingeschränkten Zugang zu wissenschaftlicher Literatur über das Internet, und die Möglichkeit, Inhalte unter bestimmten Bedingungen weiterzuverwenden. Hierdurch werden Artikel auch für Personen zugänglich, die kein entsprechendes Zeitschriften-Abonnement haben (z. B. Ärzte, die keiner akademischen Einrichtung angeschlossen sind, oder Wissenschaftler in Ländern mit eingeschränkten finanziellen Mitteln). Die Kosten für diese Modell müssen allerdings die Autoren selbst tragen (bzw. deren Institutionen), durch die Zahlung einer einmaligen Gebühr (meist um die €2.000) nach Annahme des Artikels. Beachten Sie, dass „open access“ zu publizieren nicht bedeutet, auch (rein) elektronisch zu publizieren (und umgekehrt).

Leider tummeln sich im Internet auch haufenweise unseriöse Zeitschriften (sogenannte „predatory journals“) und Herausgeber, denen einzig daran gelegen ist, über open access-Gebühren viel Geld zu verdienen. Diese Zeitschriften erkennt man daran, dass sie anhand von „phishing“ E-Mails schmeichelhafte Einladungen an potentielle Autoren verschicken und diese mit unrealistischen Angeboten versuchen zu ködern (z. B. der garantierten Veröffentlichung des Artikels innerhalb kürzester Zeit). Hiervon sollte man Abstand halten, weil solche Zeitschriften nicht wissenschaftlich arbeiten und auch plötzlich wieder verschwinden können - mitsamt der eingereichten Artikel. Seriöse Zeitschriften umschmeicheln Sie nicht mit E-Mails; das haben sie nämlich nicht nötig. Duch „predatory journals“ zu googlen kann man eine aktuelle Übersicht unseriöser Fachzeitschriften und Herausgeber finden.

\section{Was Sie tun sollten}

Denken Sie frühzeitig im Schreibprozess über eine passende Zeitschrift nach. Die Art der gewünschten Zeitschrift hat nämlich Einfluss auf den inhaltlichen Aufbau Ihres Artikels, insbesondere die Abschnitte Einleitung und Diskussion (siehe Teil 1: Anfangen zu Schreiben). 
Diskutieren Sie innerhalb Ihres Autorenteams den primären Fokus und die anvisierte Leserschaft für jeden einzelnen Artikel. Verschaffen Sie sich einen Überblick über relevante Literatur in Ihrem Fachgebiet um zu sehen, wo ähnliche Arbeiten veröffentlicht wurden. Entscheiden Sie, ob Sie vornehmlich Wissenschaftler, Gesundheitspersonal oder beide Gruppen erreichen wollen, und erstellen Sie eine Liste allgemeiner oder mehr krankheits-, bzw. inhaltsspezifischer Zeitschriften. Machen Sie eine realistische Einschätzung, wie hochrangig Sie Ihren Artikel veröffentlichen können, und bitten Sie diesbezüglich um die ehrliche Meinung erfahrener Kollegen. Fragen Sie auch nach Erfahrungen mit dem Service anvisierter Zeitschriften, z. B. nach der Qualität und Schnelligkeit des Begutachtungs- und Veröffentlichungsprozesses und der Kundenfreundlichkeit. Lesen Sie die Informationen für Autoren auf der Webseite der Zeitschrift um festzustellen, ob die Zeitschrift tatsächlich die Art von Artikel veröffentlicht, die Sie schreiben wollen.

Überlegen Sie ob es Ihnen wichtig ist, Ihren Artikel in einer Zeitschrift mit besonders hohem Impact-Faktor oder möglichst zeitnah zu veröffentlichen. Der Abstieg über die lange Leiter der Impact-Faktoren kann wegen der niedrigen Annahmeraten hochrangiger Zeitschriften lang und demotivierend sein. Wenn eine hochrangige Zeitschrift Ihren Artikel zur Begutachtung annimmt aber später doch ablehnt kann dies trotzdem nützlich sein, wenn Sie mit der Ablehnung wertvolle Gutachterkommentare erhalten (siehe Teil 12: Beantworten von Gutachterkommentaren). Insbesondere wenn Sie in einem sehr dynamischen Bereich forschen, ist die vorab Publikation auf einem pre-print Server zu überlegen. Dies fördert Transparenz und reduziert unnütze Forschung, die durch doppelte Arbeit oder nicht veröffentlichte Arbeiten entsteht. Wenn Sie in einer bestimmten Zeitschrift veröffentlichen möchten, sollten Sie diese auch lesen. Schauen Sie sich die Inhaltsverzeichnisse vorangegangener Ausgaben und einige Artikel und Leitartikel an, um sich ein Bild über die thematische Reichweite und die Präferenzen der Redakteure zu machen.

Erstellen Sie mit Ihrem Autorenteam eine Rangliste von 3-5 potentiellen Zeitschriften. Somit können Sie sich beim Schreiben auf die erste Zeitschrift Ihrer Wahl konzentrieren und sich nach deren Autorenhinweisen richten. Zudem haben Sie einen Plan für das weitere Vorgehen, sollte Ihr Artikel abgelehnt werden. 


\section{Checkliste für die Auswahl einer Zeitschrift}

- Denken Sie frühzeitig im Schreibprozess über eine passende Zeitschrift nach

- Berücksichtigen Sie bei der Auswahl folgende Merkmale der Zeitschrift:

- thematische Reichweite (passend zum primären Fokus des Artikels)

- primäre Leserschaft: allgemein vs. spezialisiert (Inhalt und Sprache der Artikels darauf abstimmen)

- Impact-Faktor (realistisch einschätzen und Annahmeraten bei Zeitplanung einkalkulieren)

- Publikationsform: traditionell (gedruckt) vs. rein elektronisch (Internet-basiert)

- Zugang: bezahlt vs. offen (open access)

- Erstellen Sie eine Rangliste von 3-5 potentiellen Zeitschriften

- Erwägen Sie die Publikation Ihres Artikels auf einem pre-print Server 